\title{
G-jitter induced mixed convection flow of a second grade fluid past an inclined stretching sheet
}

\author{
Noraihan Afiqah Rawi $^{1}$, Mohd Rijal Ilias ${ }^{1,2}$, Rahimah Mahat ${ }^{1,3}$, Zaiton Mat Isa $^{1}$, and \\ Sharidan Shafie ${ }^{1, *}$ \\ 1Department of Mathematical Sciences, Faculty of Science, Universiti Teknologi Malaysia, 81310 \\ UTM Johor Bahru, Johor, Malaysia \\ ${ }^{2}$ Department of Mathematical Sciences, Faculty of Computer and Mathematical Sciences, UiTM Shah \\ Alam, 40450 Shah Alam, Selangor, Malaysia \\ ${ }^{3}$ Universiti Kuala Lumpur Malaysian Institute of Industrial Technology, 81750 Johor Bahru, Johor, \\ Malaysia
}

\begin{abstract}
An analysis was carried out to study the mixed convection flow of a non-Newtonian second grade fluid past an inclined stretching sheet in the presence of a g-jitter effect. The transformed governing equation, consisting of coupled non-linear partial differential equations, was solved numerically using an implicit finite-difference scheme known as the Keller-box method. The flow and heat transfer characteristics in terms of velocity and temperature profiles as well as the skin friction and heat transfer coefficients influenced by the amplitude of modulation, frequency of oscillation, inclination angle and second grade parameters were presented graphically and analysed in detail. The findings revealed that the heat transfer coefficient is enhanced with an increase in the second grade parameter, whereas the opposite trend is observed for the skin friction coefficient.
\end{abstract}

\section{Introduction}

Second grade fluid, also known as viscoelastic fluid, is a non-Newtonian fluid due to the nonlinear relationship between stress and the rate of strain. In view of the increasing importance of non-Newtonian flow, especially in second grade fluids which exhibit both viscous and elastic characteristics, many researchers have studied convective heat transfer in second grade fluids using various conditions and geometries, for example: Mushtaq et al. [1], Hsiao [2], Hayat et al. [3], Hayat and Qasim [4], Olajuwon [5] and Turkyilmazoglu [6]. Recently, Hayat et al. [7] investigated the three-dimensional mixed convection flow of viscoelastic fluid past an exponentially stretching surface by taking into account the effects of thermal radiation and convective conditions.

In all of the above-mentioned papers, the second grade fluid flow over an inclined plate was not considered. It is essential to analyse the effect of an inclined plate on second grade fluid because of its great importance in the engineering, technology and manufacturing

*Corresponding author: sharidan@utm.my 
industries. In addition, Jaluria [8] indicates that the importance of buoyancy force on an inclined, continuously moving sheet not only depends on the angle of orientation, but also on the mixed convection parameter which indicates the strength of natural and forced convection flow effects. Therefore, in this paper, the unsteady mixed convection flow of second grade fluid past an inclined stretching sheet in the presence of a g-jitter effect is investigated. g-Jitter can be defined as the inertia effect due to quasi-steady, oscillatory or transient accelerations arising from crew motions and machinery vibrations in parabolic aircrafts, space shuttles or other microgravity environments. A series study on the behaviour of g-jitter with different effects and type of fluids focusing on boundary layer flow was made by Sharidan et al. [9-11]. For example, Sharidan et al. [10] studied the effect of g-jitter mixed convection on the flow and heat transfer characteristics associated with a vertical stretching sheet in a Newtonian fluid. Considering the effect of $g$-jitter in that paper, the time dependent gravitational field, $g(t)$ is defined as $g(t)=g_{0}[1+\varepsilon \cos (\pi \omega t)]$, where $g_{0}$ is the time-averaged value of the gravitational acceleration, $\varepsilon$ is a scaling parameter which gives the magnitude of the gravity modulation relative to $g_{0}, t$ is the time and $\omega$ is the frequency of oscillation of the g-jitter driven flow.

\section{Problem formulation}

Consider the unsteady mixed convection flow of a viscous and incompressible second grade fluid past an inclined stretching sheet associated with the effect of g-jitter. In this problem, the $x$-axis extends along the sheet with an inclination angle of $\alpha$ to the vertical, and the $y$-axis is normal to the sheet. The sheet is assumed to have a linear velocity of $u_{w}(x)$ in $x$-direction of the flow, and the temperature of the sheet varies linearly with distance $x$ along the sheet: $T_{w}(x)>T_{\infty}$ with $T_{w}(x)$ being the temperature of the sheet and $T_{\infty}$ being the uniform temperature of the ambient fluid. The continuous stretching sheet is assumed to have velocity and temperature in the form of $u_{w}(x)=c x$ and $T_{w}(x)=T_{\infty}+a x$, where $a$ and $c$ are constants, and $c>0$.

Under the boundary layer and Boussinesq approximations, the basic governing equation of a second grade fluid moving past an inclined stretching sheet can be written as:

$$
\begin{aligned}
\frac{\partial u}{\partial x}+\frac{\partial v}{\partial y}=0 & \\
\rho\left(\frac{\partial u}{\partial t}+u \frac{\partial u}{\partial x}+v \frac{\partial u}{\partial y}\right)= & \mu \frac{\partial^{2} u}{\partial y^{2}}+(\rho \beta) g_{0}[1+\varepsilon \cos (\pi \omega t)]\left(T-T_{\infty}\right) \cos \alpha \\
& +\alpha\left(\frac{\partial^{3} u}{\partial t \partial y^{2}}+u \frac{\partial^{3} u}{\partial x \partial y^{2}}+\frac{\partial u}{\partial x} \frac{\partial^{2} u}{\partial y^{2}}+\frac{\partial u}{\partial y} \frac{\partial^{2} u}{\partial x \partial y}+v \frac{\partial^{3} u}{\partial y^{3}}\right) \\
& \frac{\partial T}{\partial t}+u \frac{\partial T}{\partial x}+v \frac{\partial T}{\partial y}=\frac{k}{\rho C_{p}} \frac{\partial^{2} T}{\partial y^{2}}
\end{aligned}
$$

subject to the initial and boundary conditions:

$$
\begin{aligned}
& t=0: u=v=0, T=T_{\infty} \text { for any } x, y, \\
& t>0: u=u_{w}(x), v=0, T=T_{w} \text { at } y=0, \\
& u \rightarrow 0, \frac{\partial u}{\partial y} \rightarrow 0, T \rightarrow T_{\infty} \text { as } y \rightarrow \infty .
\end{aligned}
$$


where $u$ and $v$ are the velocity components along the $x$-and $y$-axes, $\rho$ is the density of the fluid, $\mu$ is the effective viscosity, $\beta$ is the thermal expansion of the fluid, $\alpha_{1}$ is the material parameter of the second grade fluid, $T$ is the temperature of the fluid, $C_{p}$ is the specific heat of the fluid at a constant pressure, and $k$ is the effective thermal conductivity of the fluid.

The complexity of the problem is reduced by introducing the similarity transformations [10]:

$$
\tau=\omega t, \eta=\left(\frac{c}{v}\right)^{1 / 2} y, \psi=(c v)^{1 / 2} x f(\tau, \eta), \theta(\tau, \eta)=\frac{\left(T-T_{\infty}\right)}{\left(T_{w}-T_{\infty}\right)},
$$

where $v$ is the effective kinematic viscosity and $\psi(x, y)$ is the non-dimensional stream function, defined in the usual way as $u=\partial \psi / \partial y$ and $v=-\partial \psi / \partial x$, and therefore equation (1) is automatically satisfied. Substitution of equation (5) into equations (2) and (3) give the following transformed governing equations:

$$
\begin{gathered}
f^{\prime \prime \prime}-\left(\left(f^{\prime}\right)^{2}-f f^{\prime \prime}\right)+K\left(2 f f^{\prime \prime \prime}-\left(f^{\prime \prime}\right)^{2}-f f^{(4)}\right)+\lambda[1+\varepsilon \cos (\pi \tau)] \theta \cos \alpha=\Omega\left(\frac{\partial f^{\prime}}{\partial \tau}-K \frac{\partial f^{\prime \prime \prime}}{\partial \tau}\right), \\
\theta^{\prime \prime}+\operatorname{Pr}\left(f \theta^{\prime}-f^{\prime} \theta\right)=\operatorname{Pr} \Omega \frac{\partial \theta}{\partial \tau},
\end{gathered}
$$

subjecting this to the boundary conditions (4), it becomes:

$$
\begin{gathered}
f=0, f^{\prime}=1, \theta=1 \text { on } \eta=0, \\
f^{\prime} \rightarrow 0, f^{\prime \prime} \rightarrow 0, \theta \rightarrow 0 \text { as } \eta \rightarrow \infty .
\end{gathered}
$$

Dimensionless parameters of present problem are,

$$
K=\frac{\alpha_{1} c}{\mu}, \operatorname{Pr}=\frac{C_{p} \mu}{k}, \Omega=\frac{\omega}{c}, \lambda=\frac{G r_{x}}{\operatorname{Re}_{x}^{2}}=\frac{g_{0} \beta\left(T_{w}(x)-T_{\infty}\right) x^{3} / v^{2}}{\left(u_{w}(x) x / v\right)^{2}}
$$

where $K$ is the dimensionless second grade parameter, $\operatorname{Pr}$ is the Prandtl number, $\Omega$ is the dimensionless frequency of oscillation and $\lambda$ is the mixed convection parameter with $G r_{x}$ and $\mathrm{Re}_{x}$ being the local Grashof number and Reynolds numbers respectively. In this study, the non-dimensional skin friction and heat transfer coefficients are obtained by:

$$
\begin{gathered}
C_{f} \operatorname{Re}_{x}^{1 / 2}=\frac{\partial^{2} f}{\partial \eta^{2}}(\tau, 0)+K\left(3 \frac{\partial f}{\partial \eta}(\tau, 0) \frac{\partial^{2} f}{\partial \eta^{2}}(\tau, 0)-f(\tau, 0) \frac{\partial^{3} f}{\partial \eta^{3}}(\tau, 0)\right), \\
N u_{x} / \operatorname{Re}_{x}^{1 / 2}=-\frac{\partial \theta}{\partial \eta}(\tau, 0) .
\end{gathered}
$$

\section{Results and discussion}

The system of equations (6) and (7) together with the boundary conditions (8) are solved numerically using the Keller-box method, an unconditionally stable implicit finite difference scheme. The effect of different values of parameters including amplitude of modulation, $\varepsilon$, frequency of oscillation, $\Omega$, second grade parameter, $K$, and inclination angle, $\alpha$, on the variation of velocity, $f^{\prime}(\eta)$, and temperature profiles, $\theta(\eta)$, as well as skin friction, $C_{f} \operatorname{Re}_{x}^{1 / 2}$, and heat transfer coefficients, $N u_{x} / \operatorname{Re}_{x}^{1 / 2}$ are analysed and discussed in detail. The comparison with the previous published work of Sharidan et al. [10] and Freidoonimehr et al. [12], as shown in table 1, reveals a good agreement, which validates the use of the present scheme. 
Table 1. Comparison results of the heat transfer rate, $\theta^{\prime}(\eta)$ for $\varepsilon=\Omega=\lambda=\alpha=K=0$ and various values of $\operatorname{Pr}$.

\begin{tabular}{|c|c|c|c|}
\hline $\operatorname{Pr}$ & Sharidan et al. $[10]$ & Freidoonimehr et al. $[12]$ & Present \\
\hline 0.72 & 0.8086 & 0.8086 & 0.8086 \\
\hline 1.0 & 1.0000 & 1.0000 & 1.0000 \\
\hline 3.0 & 1.9238 & 1.9237 & 1.9238 \\
\hline 10.0 & 3.7225 & 3.7207 & 3.7226 \\
\hline
\end{tabular}

Figures 1 and 2 depict the variation of $f^{\prime}(\eta)$ and $\theta(\eta)$ for fixed values of $\varepsilon=0.3$ and $\Omega=0.2$ under the influence of the second grade parameter, $K$, and an inclination angle, $\alpha$. Figure 1 shows that an increase in $K$ leads to an increase of $f^{\prime}(\eta)$, while the opposite behaviour is observed for $\theta(\eta)$. Based on Equation (9), dimensionless second grade parameter, $K$ is inversely proportional to the dynamic viscosity of the fluid; thus, an increase in $K$ corresponds to a decrease in fluid viscosity, which will serve to accelerate the flow. Further, it is observed from figure 2 that the variation of $f^{\prime}(\eta)$ decreases with an increases in $\alpha$, whereas the contradict behaviour is observed for $\theta(\eta)$. These behaviours are consistent with the physical expectation that increasing $\alpha$ reduces the buoyancy force, which in turn lowers the fluid velocity resulting in a higher fluid temperature
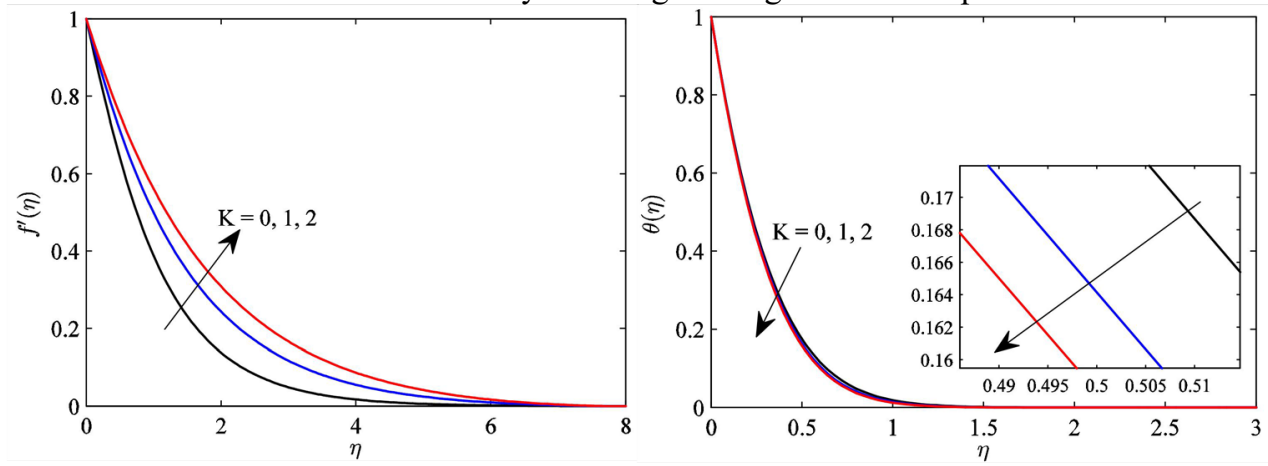

Fig. 1. Variation of $f^{\prime}(\eta)$ and $\theta(\eta)$ for various values of $K$.
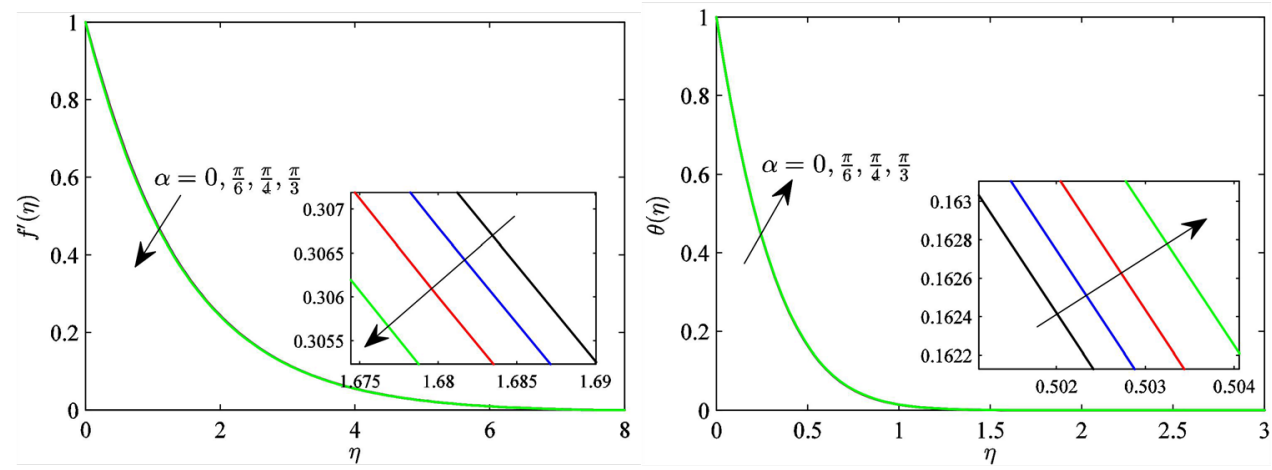

Fig. 2. Variation of $f^{\prime}(\eta)$ and $\theta(\eta)$ for various values of $\alpha$.

Figures 3 to 5 illustrate the variation of $C_{f} \operatorname{Re}_{x}^{1 / 2}$ and $N u_{x} / \mathrm{Re}_{x}^{1 / 2}$ with $\tau$ for different values of $\varepsilon, \Omega, \mathrm{K}$ and $\alpha$. It is observed from figures 3 and 4 that the values of $C_{f} \mathrm{Re}_{x}^{1 / 2}$ 
and $N u_{x} / \operatorname{Re}_{x}^{1 / 2}$ increase up to the maximum values for the increasing values of $\varepsilon$, and then decreases with the dimensionless time, $\tau$. Furthermore, it is also noticed that the amplitude of the peak response $C_{f} \operatorname{Re}_{x}^{1 / 2}$ and $N u_{x} / \operatorname{Re}_{x}^{1 / 2}$ decreases with increasing $\Omega$. Comparisons between figures 3 and 4 also reveal that increasing values of $K$ lead to an increase in the value of $N u_{x} / \operatorname{Re}_{x}^{1 / 2}$, but a decrease in $C_{f} \operatorname{Re}_{x}^{1 / 2}$. From figure 5, it can be seen that both $C_{f} \operatorname{Re}_{x}^{1 / 2}$ and $N u_{x} / \operatorname{Re}_{x}^{1 / 2}$ decrease as the values of $\alpha$ increase from 0 to $\frac{\pi}{3}$.
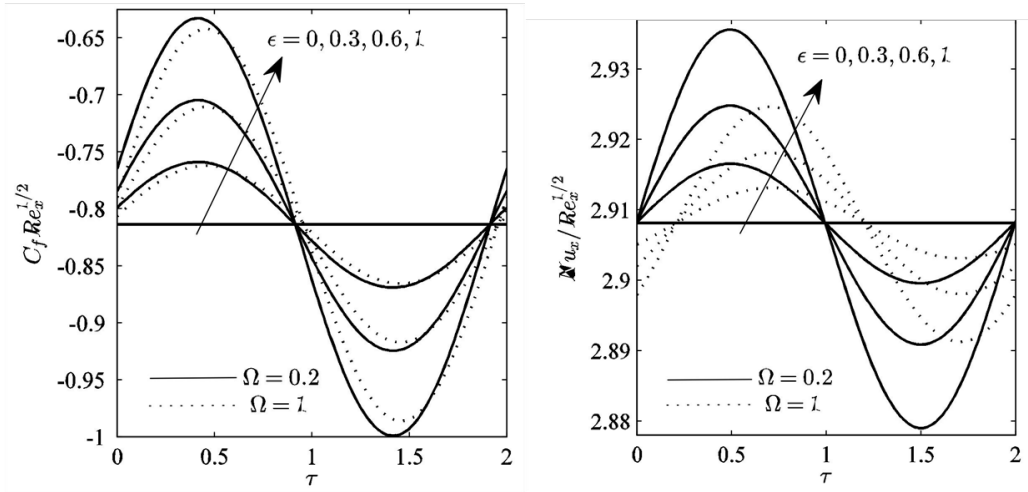

Fig. 3. Variation of $C_{f} \operatorname{Re}_{x}^{1 / 2}$ and $N u_{x} / \mathrm{Re}_{x}^{1 / 2}$ for various values of $\varepsilon$ and $\Omega$ when $K=0$.
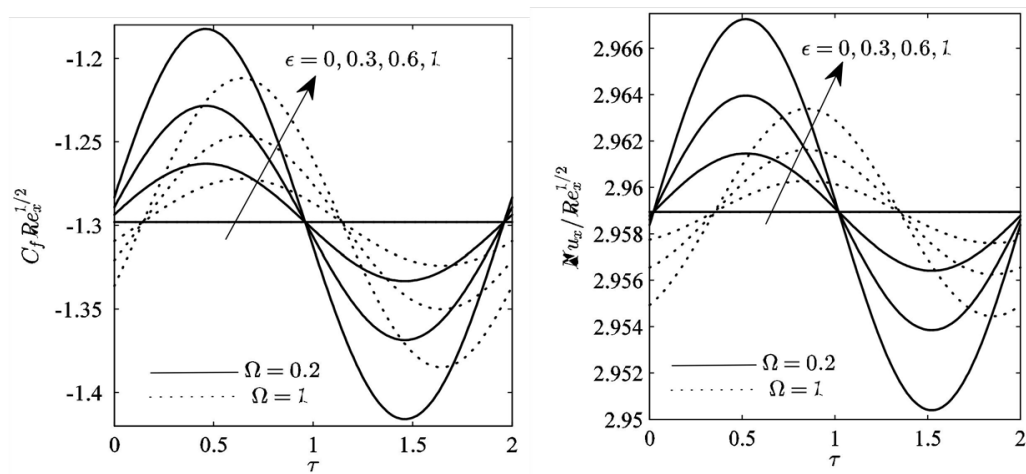

Fig. 4. Variation of $C_{f} \operatorname{Re}_{x}^{1 / 2}$ and $N u_{x} / \operatorname{Re}_{x}^{1 / 2}$ for various values of $\varepsilon$ and $\Omega$ when $K=1$.
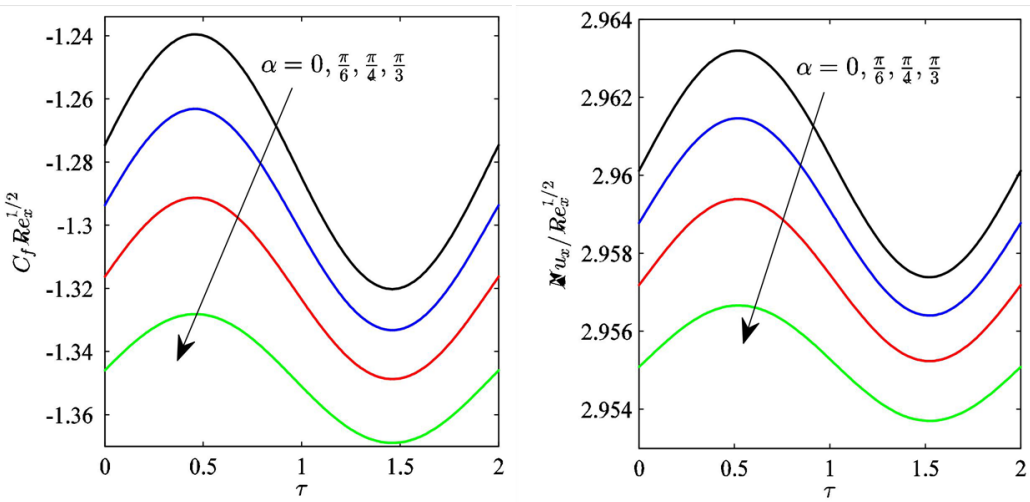

Fig. 5. Variation of $C_{f} \operatorname{Re}_{x}^{1 / 2}$ and $N u_{x} / \operatorname{Re}_{x}^{1 / 2}$ for various values of $\alpha$ when $K=1$. 


\section{Conclusion}

The problem of the mixed convection flow of second grade fluid past an inclined stretching sheet in the presence of a g-jitter effect has been investigated. Numerical results reveal that the inclination angle has a retarding effect on the flow field and enhances the temperature field, whereas the opposite effect is observed for the second grade parameter. Furthermore, the enhancement of heat transfer can be clearly seen under the influence of the second grade parameter.

\section{Acknowledgement}

The authors would like to acknowledge Ministry of Higher Education (MOHE) and Research Management Centre-UTM for the financial support through vote numbers $13 \mathrm{H} 74$ and $15 \mathrm{H} 80$ for this research.

\section{References}

1. Mushtaq M, Asghar S and Hossain MA (2007). Heat Mass Transfer 431049

2. Hsiao KL (2007). Appl Thermal Eng 271895

3. Hayat T, Mustafa M and Pop I (2010). Commun. Nonlinear Sci. Numerical Simulation 151183

4. Hayat T and Qasim M (2011). Int J Numer Methods in Fluids 66820

5. Olajuwon BI (2011). Int Commun Heat Mass Transfer 38377

6. Turkyilmazoglu M (2013). Int J Mechanical Sciences 77263

7. Hayat T, Anwar MS, Farooq M and Alsaedi A (2015). PloS One 101

8. Jaluria Y (1980). Natural Convection: Heat and Mass Transfer (Pergamon Press Oxford)

9. Sharidan S, Amin N and Pop I (2005). Int Commun Heat Mass Transfer 32657

10. Sharidan S, Amin N and Pop I (2006). Microgravity-Sci Tech 185

11. Sharidan S, Amin N and Pop I (2007). Mech Research Commun 34115

12. Freidoonimehr N, Rashidi MM and Mahmud S (2015). Int. J. Thermal Sciences 87136 